\title{
Janadolide, a Cyclic Polyketide-Peptide Hybrid Possessing a $t$-butyl Group from an Okeania sp. Marine Cyanobacterium
}

Hidetoshi Ogawa, ${ }^{\dagger}$ Arihiro Iwasaki, ${ }^{\dagger}$ Shinpei Sumimoto, ${ }^{\dagger}$ Yuki Kanamori, ${ }^{\dagger}$ Osamu Ohno, ${ }^{\star}$ Masato Iwatsuki, ${ }^{\S, \perp}$ Aki Ishiyama,${ }^{\S, \perp}$ Rei Hokari, ${ }^{\S}$ Kazuhiko Otoguro, ${ }^{\S}$ Satoshi Ōmura ${ }^{\S}$ and Kiyotake Suenaga $*,+$

$\dagger$ Department of Chemistry, Keio University, 3-14-1 Hiyoshi, Kohoku-ku, Yokohama, Kanagawa 223-8522, Japan.

† Department of Chemistry and Life Science, Kogakuin University, Nakano 2665-1, Hachioji, Tokyo 192-0015, Japan.

$\S$ Research Center for Tropical Diseases, Kitasato Institute for Life Sciences, Kitasato University, 5-9-1, Shirokane, Minato-ku, Tokyo 108-8641, Japan.

$\perp$ Graduate School of Infection Control Sciences, Kitasato University, 5-9-1, Shirokane, Minato-ku, Tokyo 108-8641, Japan.

Corresponding Author's E-mail Address: suenaga@chem.keio.ac.jp 
NMR spectra of janadolide (1) in $\mathrm{C}_{6} \mathrm{D}_{6}$

${ }^{1} \mathrm{H}$ NMR spectra of $\mathbf{2 a}, \mathbf{2} \mathbf{b}, \mathbf{3 a}, \mathbf{3} \mathbf{b}$ and the authentic sample of

(R)-PGME amide of (S)-2-hydroxy-3,3-dimethylbutanoic acid

Table S1. Detailed NMR spectral data of janadolide (1) in $\mathrm{C}_{6} \mathrm{D}_{6}$

Determination of the absolute configuration of C-2 in the polyketide moiety

Determination of the absolute configuration of the amino acid moieties in janadolide (1) S16 - S19

NMR spectra of janadolide (1) in $\mathrm{CDCl}_{3}$

S20 - S22

Molecular modeling based on NMR data

S23 - S24

Table S2. Detailed NMR spectral data of janadolide (1) in $\mathrm{CDCl}_{3}$

S25

Table S3. Constraints for molecular modeling of janadolide (1)

Evaluation of the biological activity of janadolide (1)

S27

Identification of the marine cyanobacterium

S28

Reference for the supporting information 


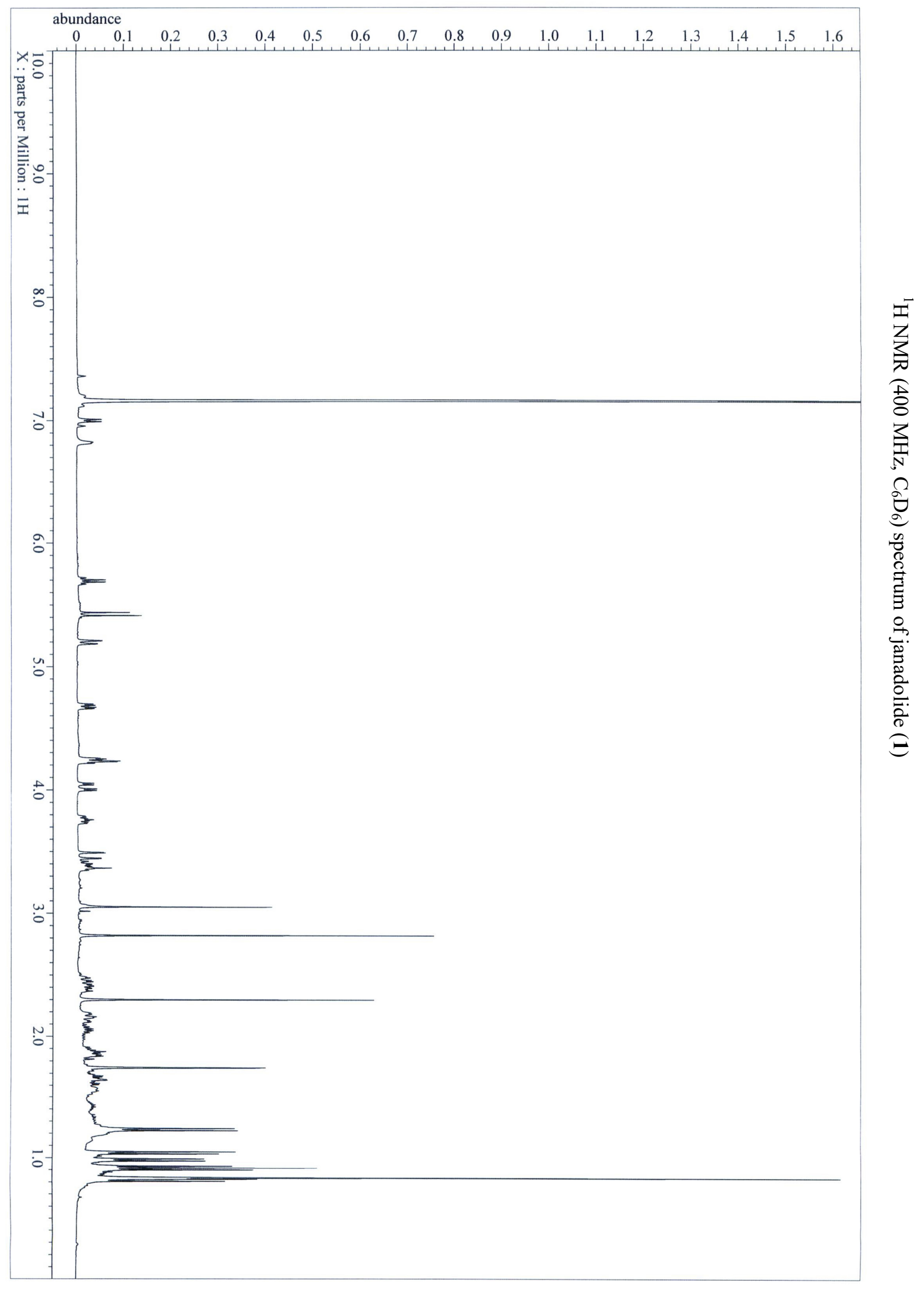




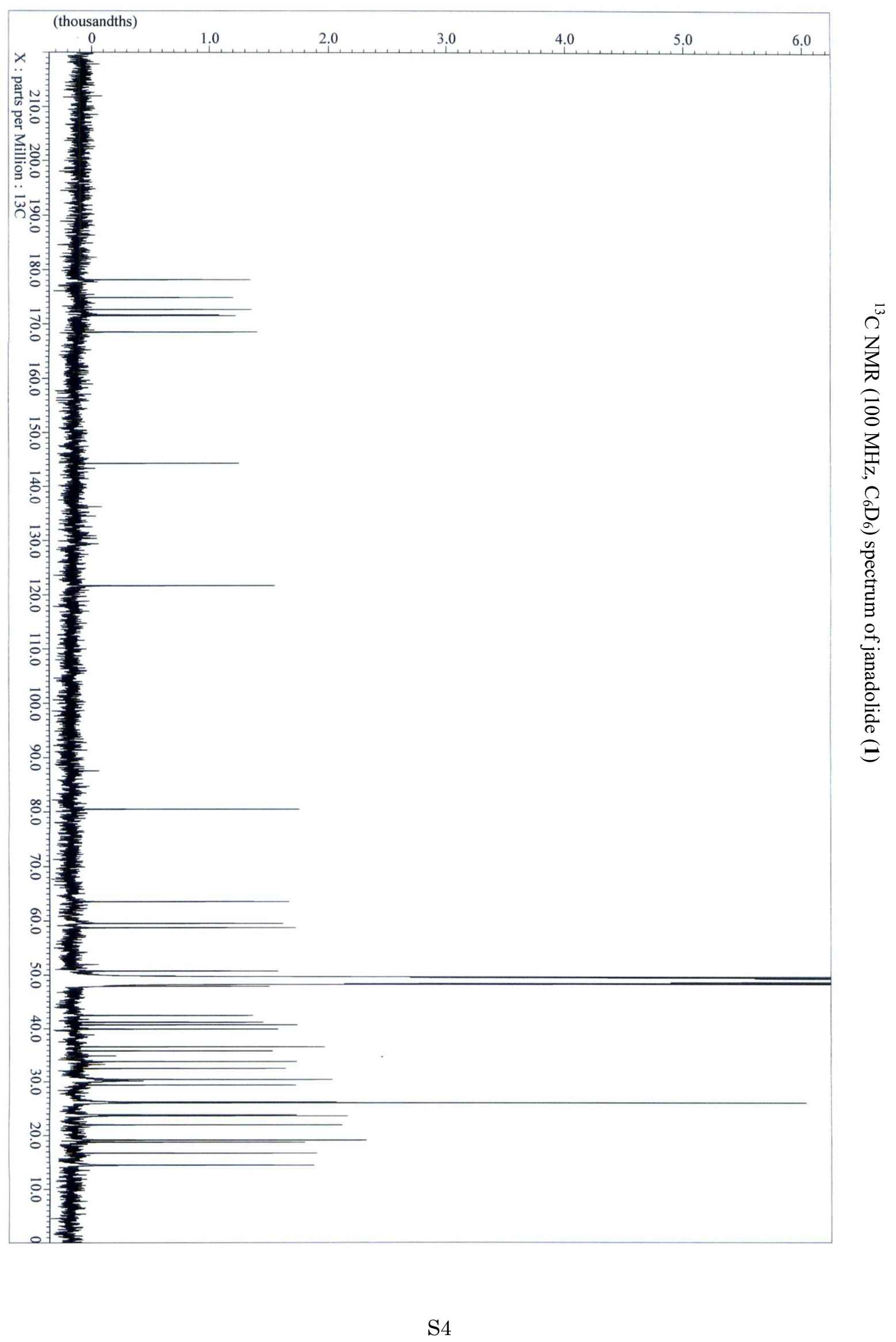




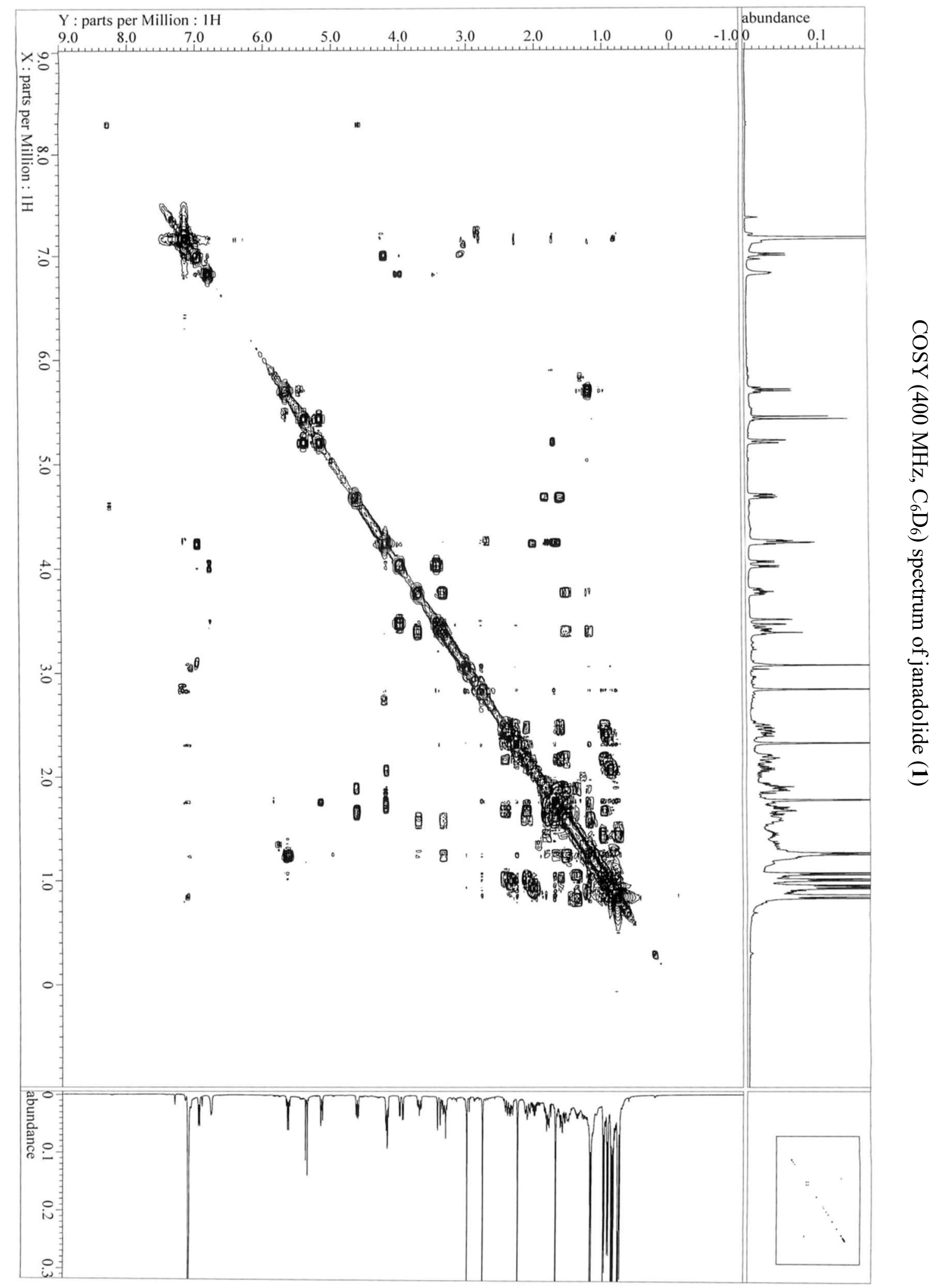




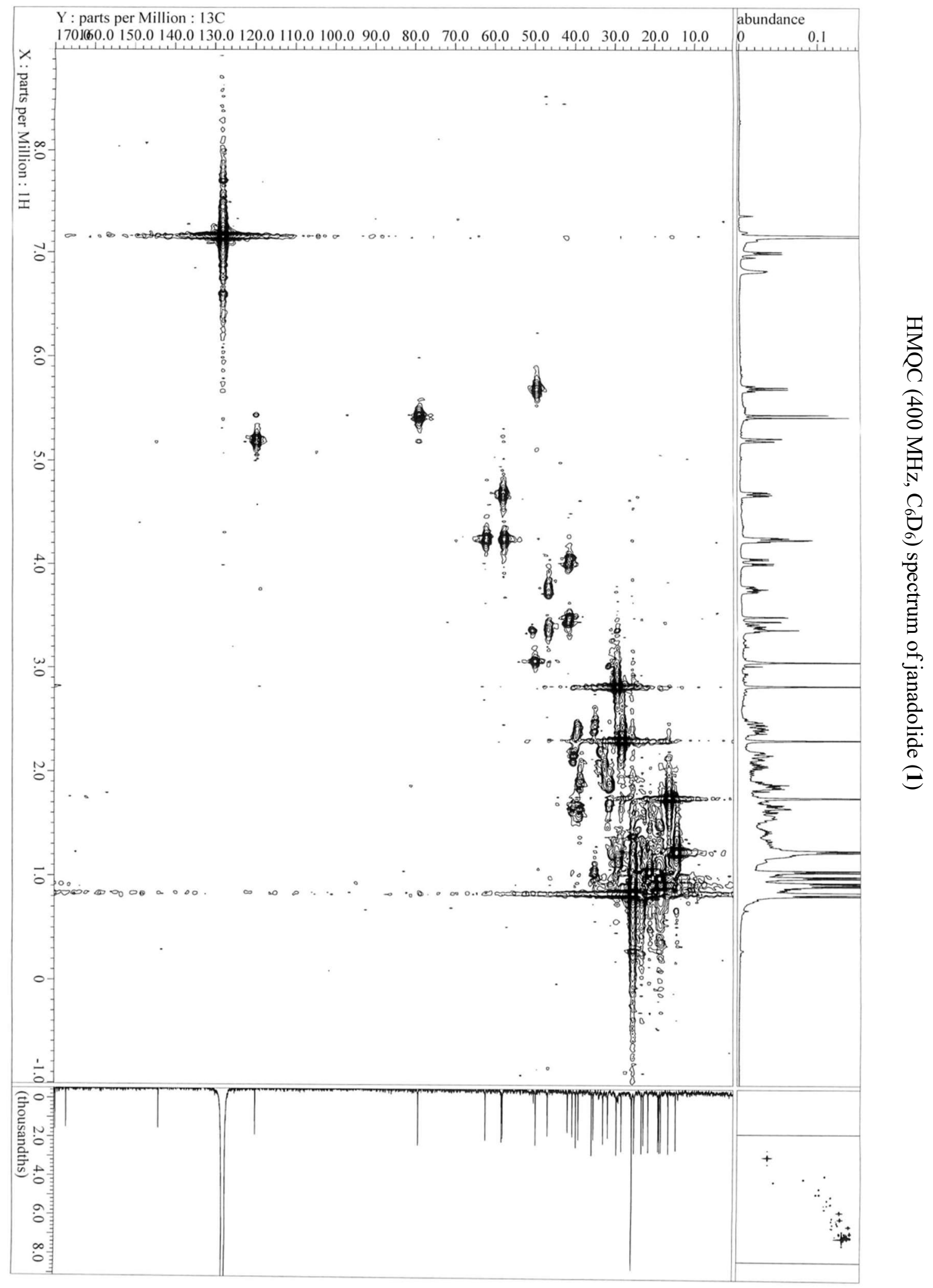




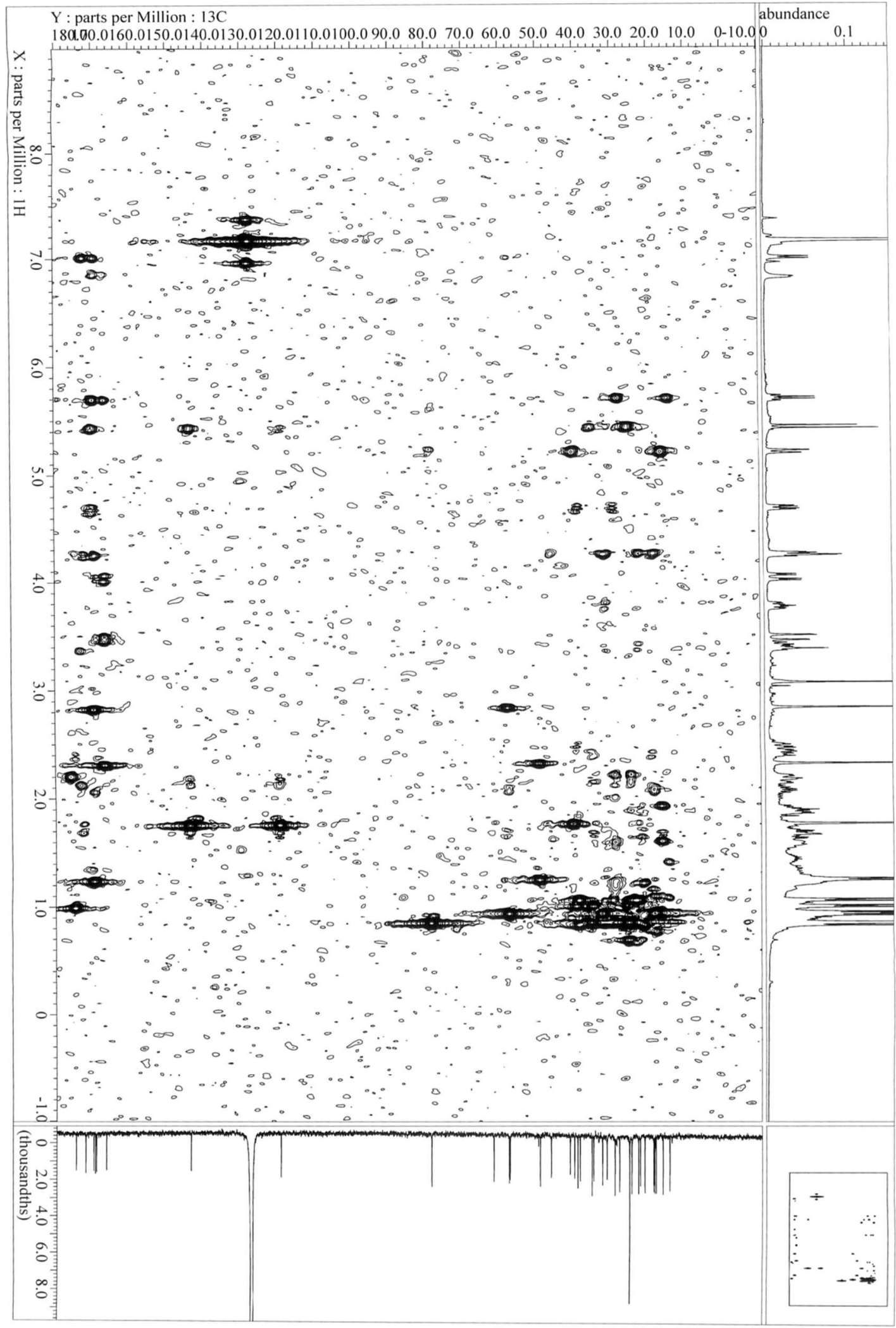




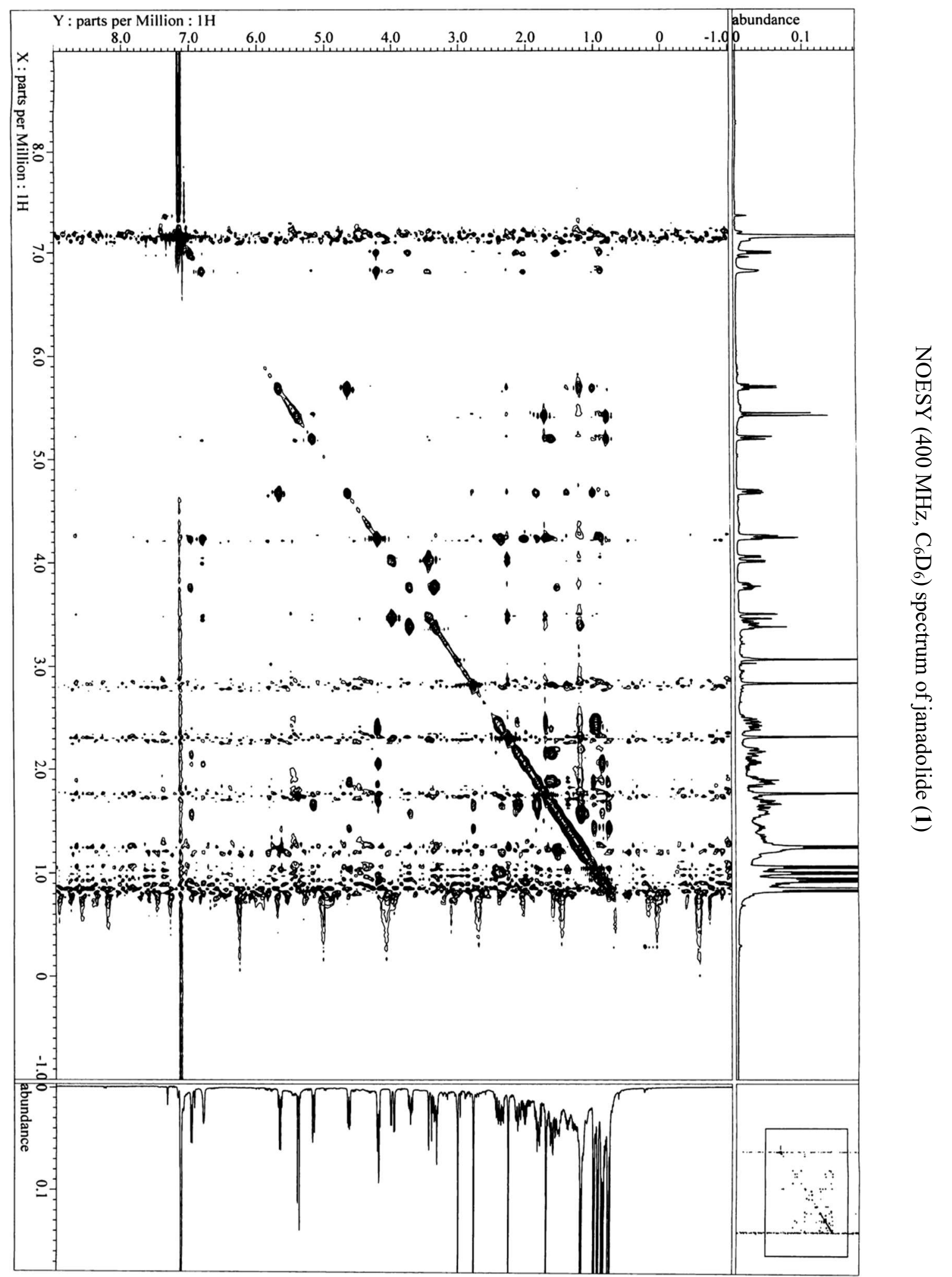




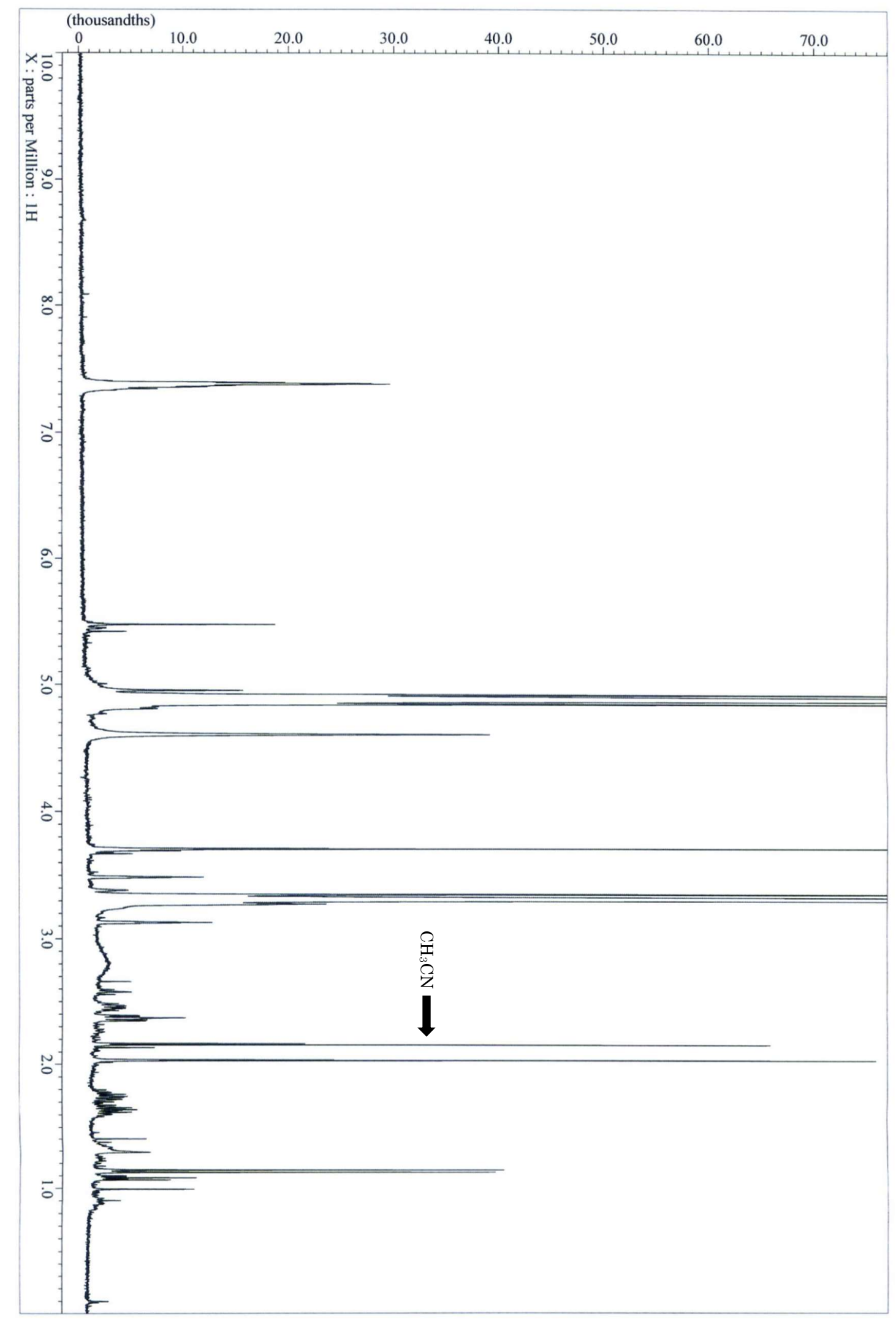

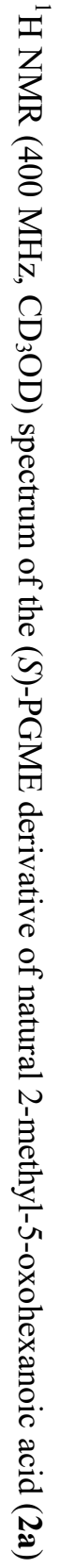




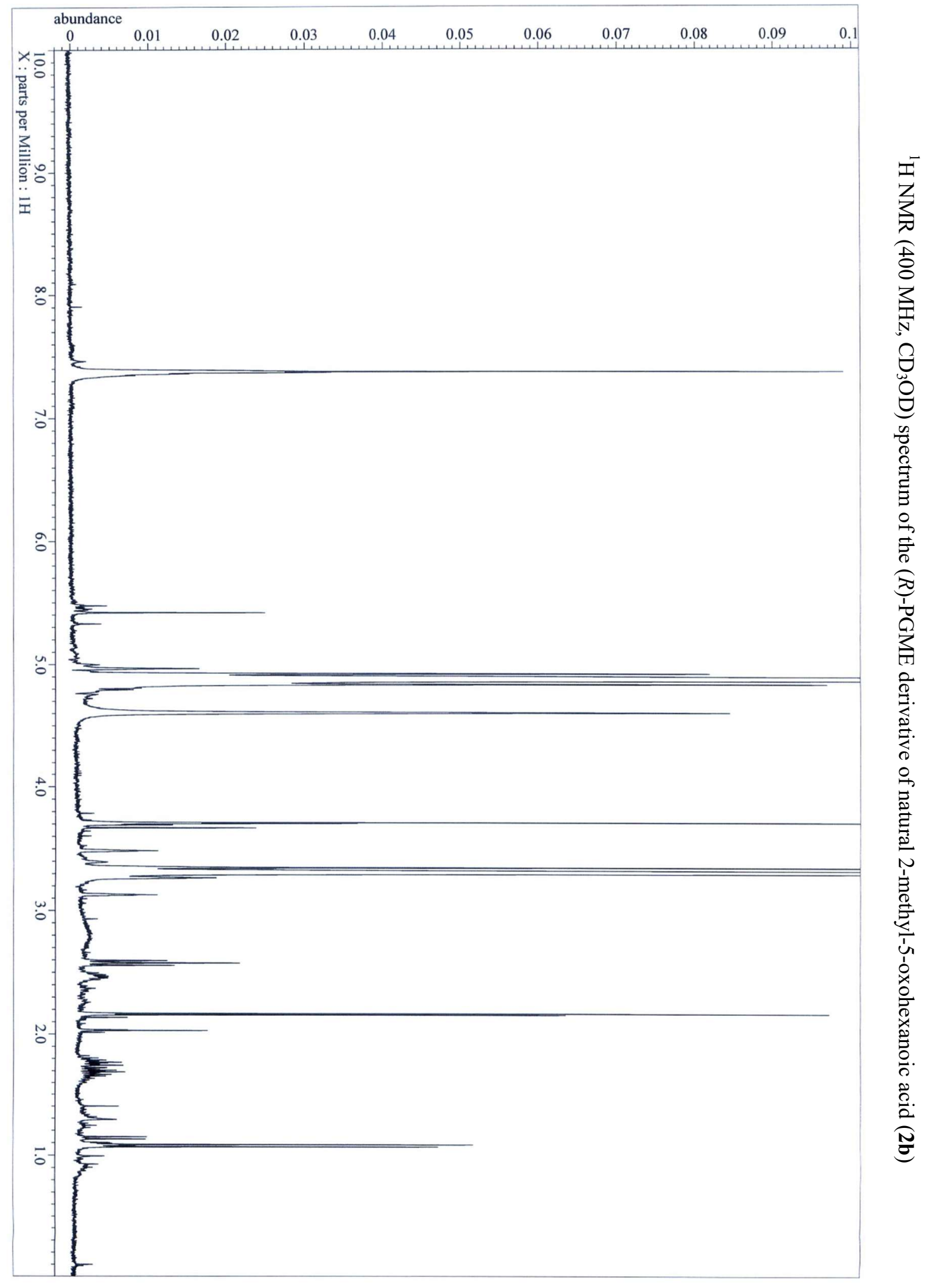




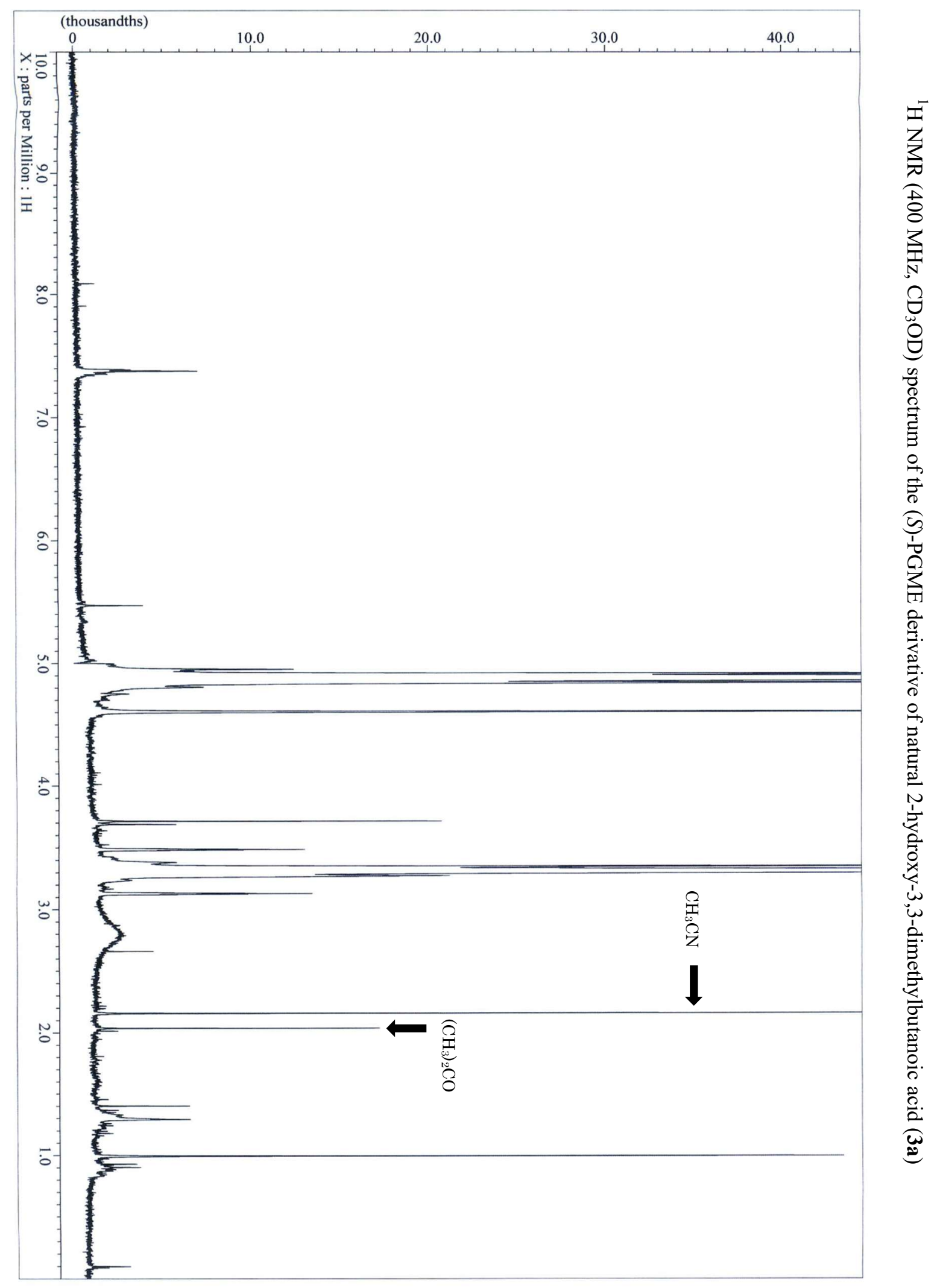




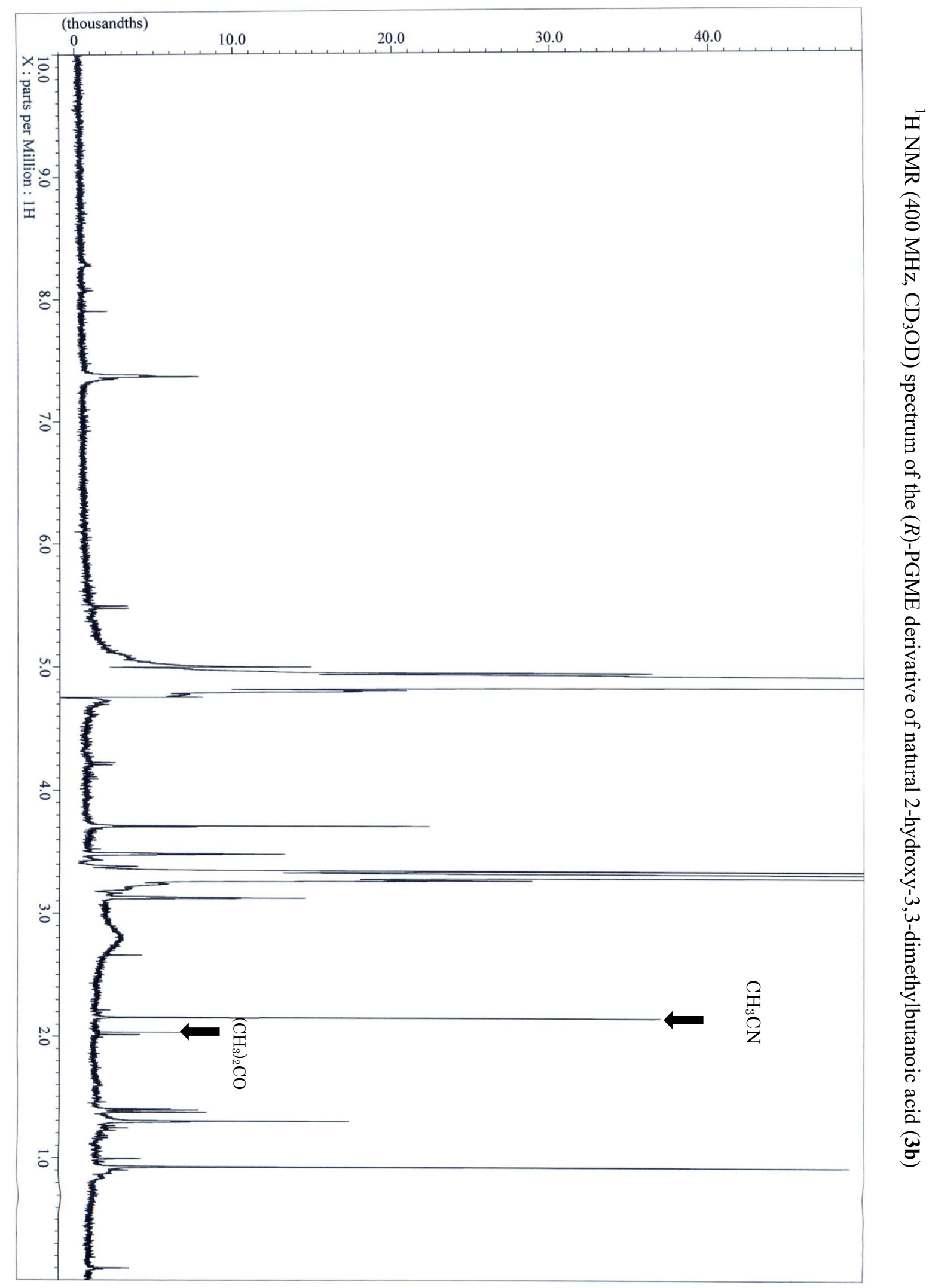




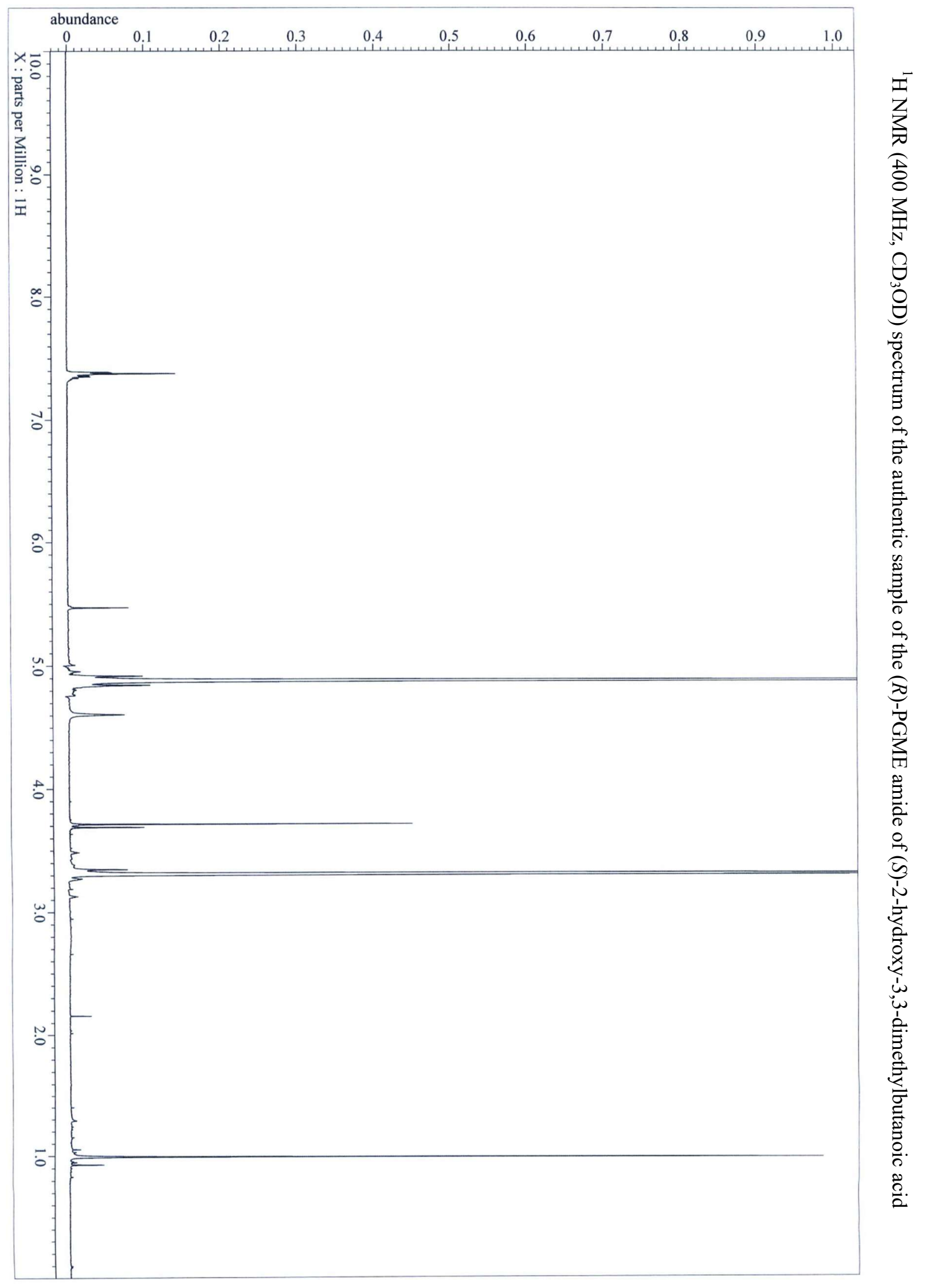


Table S1. Detailed NMR spectral data of janadolide (1) in $\mathrm{C}_{6} \mathrm{D}_{6}$

\begin{tabular}{|c|c|c|c|c|c|c|}
\hline Unit & Position & $\delta_{\mathrm{C}^{\mathrm{a}}}^{\mathrm{a}}$ & $\delta_{\mathrm{H}^{\mathrm{b}}}^{\mathrm{b}}(J$ in $\mathrm{Hz})$ & COSY & $\mathrm{HMBC}(\mathrm{H} \rightarrow \mathrm{C})$ & NOESY \\
\hline \multirow[t]{8}{*}{ Pro } & 1 & 172.7, C & & & & \\
\hline & 2 & $62.4, \mathrm{CH}$ & $4.236, \mathrm{~m}$ & $3 a, 3 b$ & $1,3,4,5,1$ (fatty acid) & 3a, 3b, 2 (Fatty acid), 10 (Fatty acid) \\
\hline & $3 a$ & $31.8, \mathrm{CH}_{2}$ & $1.89, \mathrm{~m}$ & $2,3 b, 4 a, 4 b$ & 4 & 2, 4b, 2 (Val), 5 (Val) \\
\hline & $3 b$ & & $1.72, \mathrm{~m}$ & $2,3 a, 4 a, 4 b$ & 1 & 2,2 (Val) \\
\hline & $4 a$ & $22.9, \mathrm{CH}_{2}$ & $1.56, \mathrm{~m}$ & $3 a, 3 b, 4 b, 5 a, 5 b$ & & $4 b, 5 a$ \\
\hline & $4 \mathrm{~b}$ & & $1.234, \mathrm{~m}$ & $3 a, 3 b, 4 a, 5 a, 5 b$ & & $4 a, 3 a, 5 b$ \\
\hline & $5 \mathrm{a}$ & $46.8, \mathrm{CH}_{2}$ & 3.76 , ddd $(12.0,8.5,3.5)$ & $4 a, 4 b, 5 b$ & 3 & $4 a, 5 b$ \\
\hline & $5 b$ & & $3.39, \mathrm{~m}$ & $4 a, 4 b, 5 a$ & 4 & $4 b, 5 a$ \\
\hline \multirow[t]{6}{*}{ Val } & 1 & $169.8, \mathrm{C}$ & & & & \\
\hline & 2 & $58.0, \mathrm{CH}$ & $4.241, \mathrm{~m}$ & $3, \mathrm{NH}$ & $1,3,5,1$ (Pro) & $3,4,5,3 \mathrm{a}$ (Pro), 3b (Pro) \\
\hline & 3 & $33.0, \mathrm{CH}$ & $2.05, \mathrm{~m}$ & $2,4,5$ & $1,2,4,5$ & $2,4,5$ \\
\hline & 4 & $19.0, \mathrm{CH}_{3}$ & $0.92, d(6.6)$ & 3 & $2,3,5$ & $2,3,5$ \\
\hline & 5 & $18.5, \mathrm{CH}_{3}$ & $0.91, \mathrm{~d}(6.7)$ & 3 & $2,3,4$ & $2,3,4,3 \mathrm{a}$ (Pro) \\
\hline & NH & & $7.00, \mathrm{~d}(6.9)$ & 2 & 1, 1 (Pro) & \\
\hline \multirow[t]{4}{*}{ Gly } & 1 & 167.1, C & & & & \\
\hline & $2 \mathrm{a}$ & $41.8, \mathrm{CH}_{2}$ & $4.02, \mathrm{dd}(18.3,5.4)$ & $2 b, \mathrm{NH}$ & 1 & 2b, $N$-Me $(N$-Me-Ala $)$ \\
\hline & $2 b$ & & 3.47 , dd $(18.3,2.1)$ & 2a, NH & 1 & 2a, $N$-Me $(N$-Me-Ala), \\
\hline & NH & & 6.82, dd $(5.4,2.1)$ & $2 a, 2 b$ & 1 (Val) & \\
\hline \multirow[t]{4}{*}{$N$-Me-Ala } & 1 & $170.1, \mathrm{C}$ & & & & \\
\hline & 2 & $49.8, \mathrm{CH}$ & $5.69, \mathrm{q}(6.8)$ & 3 & 1, 3, $N$-Me, 1 (Gly) & $3, N$-Me, $2(N$-Me-Leu), 6 ( $N$-Me-Leu) \\
\hline & 3 & $14.9, \mathrm{CH}_{3}$ & $1.232, \mathrm{~d}(6.8)$ & 2 & 1,2 & $2, N$-Me \\
\hline & $N$-Me & $28.5, \mathrm{CH}_{3}$ & $2.30, \mathrm{~s}$ & & 2, 1 (Gly) & 2, 3, 2a (Gly), 2b (Gly) \\
\hline \multirow[t]{8}{*}{$N$-Me-Leu } & 1 & 170.6, C & & & & \\
\hline & 2 & $58.2, \mathrm{CH}$ & $4.68, \mathrm{dd}(10.7,4.3)$ & $3 a, 3 b$ & $1,3, N-\mathrm{Me}$ & 3a, 3b, 4, 6, 2 (N-Me-Ala) \\
\hline & $3 a$ & $39.1, \mathrm{CH}_{2}$ & $1.87, \mathrm{~m}$ & $2,3 \mathrm{~b}, 4$ & 4 & $2,3 b, 5,6$ \\
\hline & $3 b$ & & $1.64, \mathrm{~m}$ & $2,3 \mathrm{a}$ & 1 & 2, 3a, $5, N-\mathrm{Me}$ \\
\hline & 4 & $25.2, \mathrm{CH}$ & $1.42, \mathrm{~m}$ & $3 a, 5,6$ & & $2,5,6, N-\mathrm{Me}$ \\
\hline & 5 & $23.4, \mathrm{CH}_{3}$ & $0.82, \mathrm{~d}(6.7)$ & 4 & 3,6 & $3 \mathrm{a}, 3 \mathrm{~b}, 4,6, N-\mathrm{Me}$ \\
\hline & 6 & $21.7, \mathrm{CH}_{3}$ & $1.04, \mathrm{~d}(6.5)$ & 4 & 3,5 & $2,3 \mathrm{a}, 4,5,2(N$-Me-Ala $)$ \\
\hline & $N$-Me & $29.7, \mathrm{CH}_{3}$ & $2.82, \mathrm{~s}$ & & 2, 1 ( $N$-Me-Ala) & $3 b, 4,5$ \\
\hline \multirow[t]{13}{*}{ Polyketide moity } & 1 & $175.2, \mathrm{C}$ & & & & \\
\hline & 2 & $39.7, \mathrm{CH}$ & $2.40, \mathrm{~m}$ & 10 & $1,3,10$ & $4 \mathrm{~b}, 10,2$ (Pro) \\
\hline & $3 a$ & $35.4, \mathrm{CH}_{2}$ & $2.43, \mathrm{~m}$ & $3 \mathrm{~b}, 4 \mathrm{a}, 4 \mathrm{~b}$ & 2 & $3 b, 11$ \\
\hline & $3 b$ & & $1.01, \mathrm{~m}$ & $2,3 a, 4 a, 4 b$ & & $3 a$ \\
\hline & $4 a$ & $40.6, \mathrm{CH}_{2}$ & $2.15, \mathrm{~m}$ & $3 a, 3 b, 4 b$ & $3,5,6$ & $4 b, 11$ \\
\hline & $4 b$ & & $1.66, \mathrm{~m}$ & $4 a, 3 a, 3 b$ & 3,11 & $2,4 \mathrm{a}, 6$ \\
\hline & 5 & $144.3, \mathrm{C}$ & & & $4,7,11$ & \\
\hline & 6 & $120.0, \mathrm{CH}$ & $5.20, \mathrm{dq}(10.1,0.9)$ & 7,11 & $4,7,11$ & $4 \mathrm{~b}, 9$ \\
\hline & 7 & $79.3, \mathrm{CH}$ & $5.43, \mathrm{~d}(10.1)$ & 6 & $5,6,8,9,1(N$-Me-Leu $)$ & 9,11 \\
\hline & 8 & $35.8, \mathrm{C}$ & & & 7,9 & \\
\hline & 9 & $25.8, \mathrm{CH}_{3} \times 3$ & $0.83, \mathrm{~s}$ & & 7,8 & $6,7,11$ \\
\hline & 10 & $19.2, \mathrm{CH}_{3}$ & $0.98, \mathrm{~d}(6.7)$ & 2 & $1,2,3$ & 2, 2 (Pro) \\
\hline & 11 & $16.7, \mathrm{CH}_{3}$ & $1.75, \mathrm{~d}(0.9)$ & 6 & $4,5,6$ & $3 \mathrm{a}, 4 \mathrm{a}, 7,9$ \\
\hline
\end{tabular}

${ }^{\mathrm{a}}$ Measured at $100 \mathrm{MHz} .{ }^{\mathrm{b}}$ Measured at $400 \mathrm{MHz}$. 
Determination of the absolute configuration of $\mathrm{C}-2$ in the polyketide moiety.

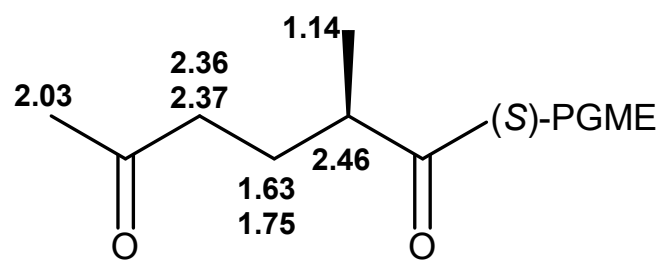

${ }^{1} \mathrm{H}$ NMR chemical shifts of (S)-PGME amide (2a)

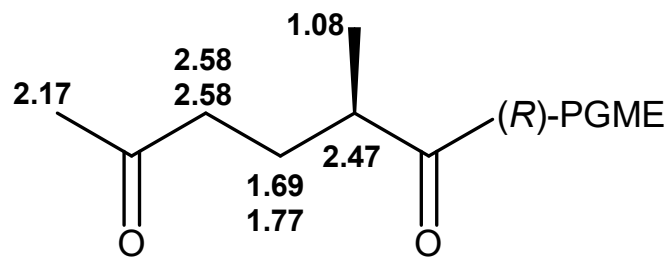

${ }^{1} \mathrm{H}$ NMR chemical shifts of $(R)$-PGME amide (2b)

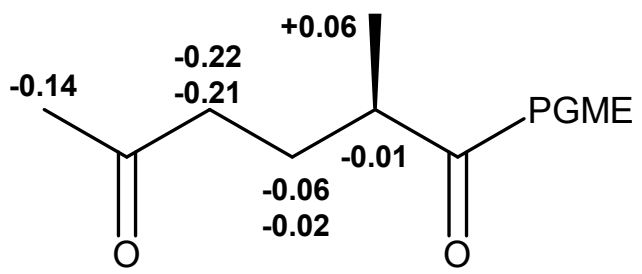

$\Delta \delta\left(\delta(S)\right.$-PGME $\left.-\delta_{(R)-P G M E}\right)$

Chemical shift differences $(\Delta \delta)$ between the PGME amides 
Determination of the absolute configuration of amino acid components by Chiral HPLC analyses.

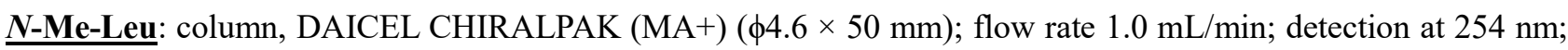
solvent, $2.0 \mathrm{mM} \mathrm{CuSO}_{4}$.

$\mathrm{t}_{R}(\min )$ : Authentic samples: $N$-Me-L-Leu (16.3), $N$-Me-D-Leu (10.3).

$N$-Me-Leu from natural 1 (16.3).

$N$-Me-D-Leu

$N$-Me-L-Leu

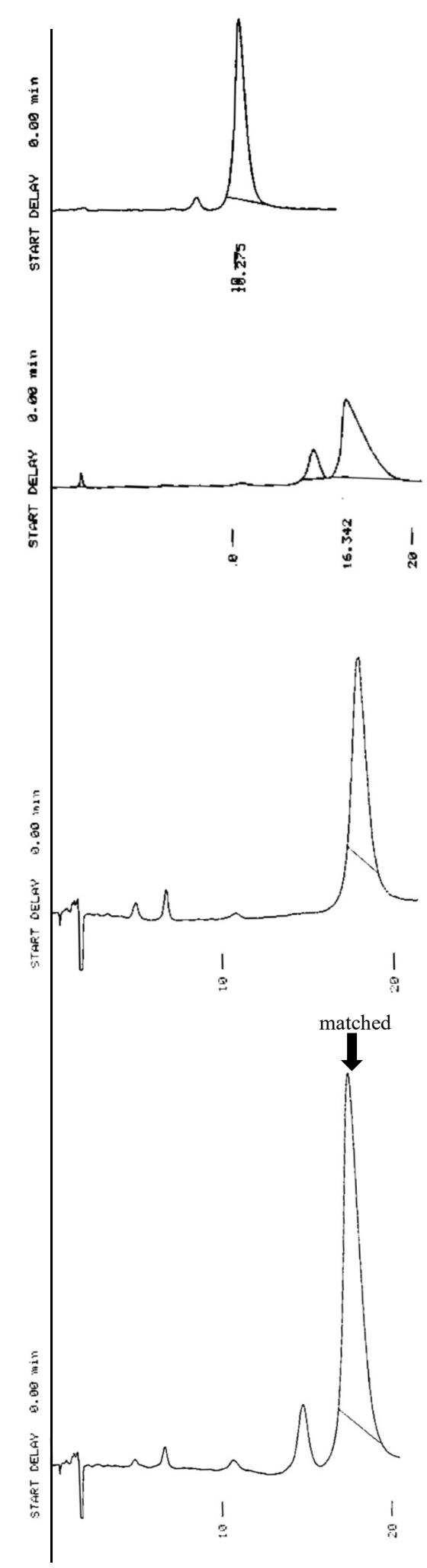

$N$-Me-Leu from natural

$N$-Me-Leu from natural 1 with $N$-Me-L-Leu 
Pro: column, DAICEL CHIRALPAK $(\mathrm{MA}+)(\phi 4.6 \times 50 \mathrm{~mm})$; flow rate $1.0 \mathrm{~mL} / \mathrm{min}$; detection at $254 \mathrm{~nm}$; solvent, $2.0 \mathrm{mM} \mathrm{CuSO}_{4}$.

$\mathrm{t}_{R}$ (min): Authentic samples: L-Pro (4.8), D-Pro (2.7).

Pro from natural 1 (4.8).

D-Pro

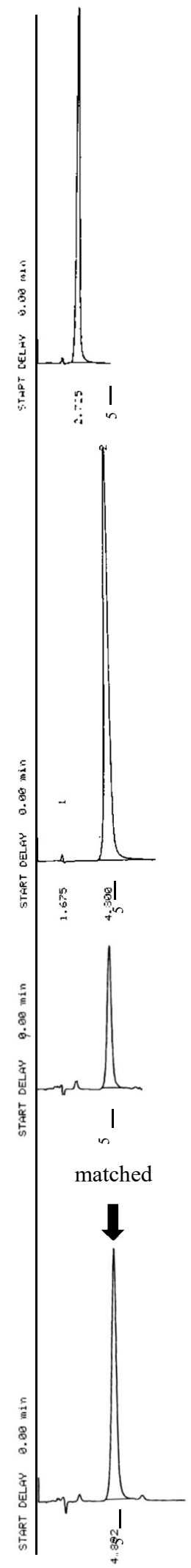

Pro from natural

Pro from natural 1 with L-Pro

L-Pro 
Val: column, DAICEL CHIRALPAK $(\mathrm{MA}+)(\phi 4.6 \times 50 \mathrm{~mm})$; flow rate $1.0 \mathrm{~mL} / \mathrm{min}$; detection at $254 \mathrm{~nm}$; solvent, $2.0 \mathrm{mM} \mathrm{CuSO}_{4}$.

$\mathrm{t}_{R}$ (min): Authentic samples: L-Val (6.0), D-Val (3.4).

Val from natural $1(6.0)$.

D-Val

L-Val

Val from natural

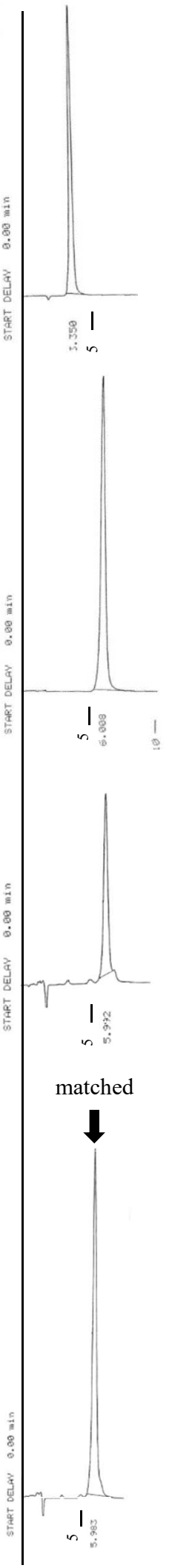

Val from natural 1 with L-Val 


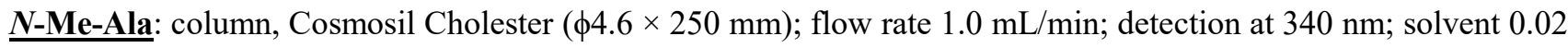
$\mathrm{M} \mathrm{NaOAc}$ aq/MeOH (45/55).

$\mathrm{t}_{R}(\mathrm{~min})$ : Marfey derivatives of authentic samples: $N$-Me-L-Ala (12.7), $N$-Me-D-Ala (21.8).

Marfey derivative of $N$-Me-Ala from natural 1 (12.7).

Marfey's derivative of $N$-Me-D-Ala

Marfey's derivative of $N$-Me-L-Ala

Marfey's derivative of

$N$-Me-Ala from natural

Marfey's derivative of

$N$-Me-Ala from natural 1

with Marfey's derivative of $N$-Me-L-Ala

Marfey reagents only

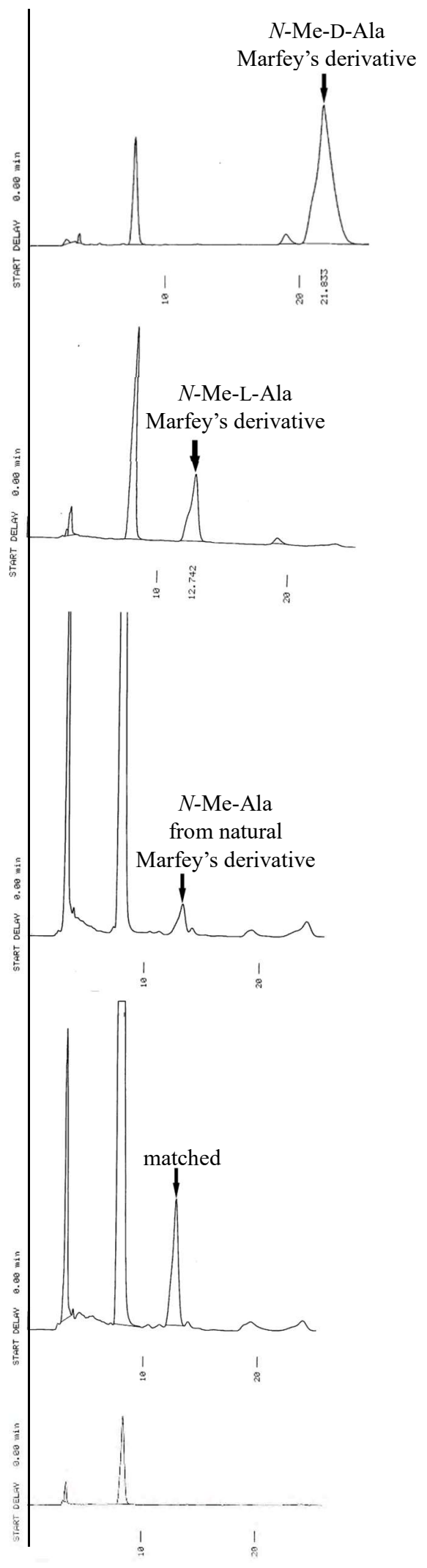




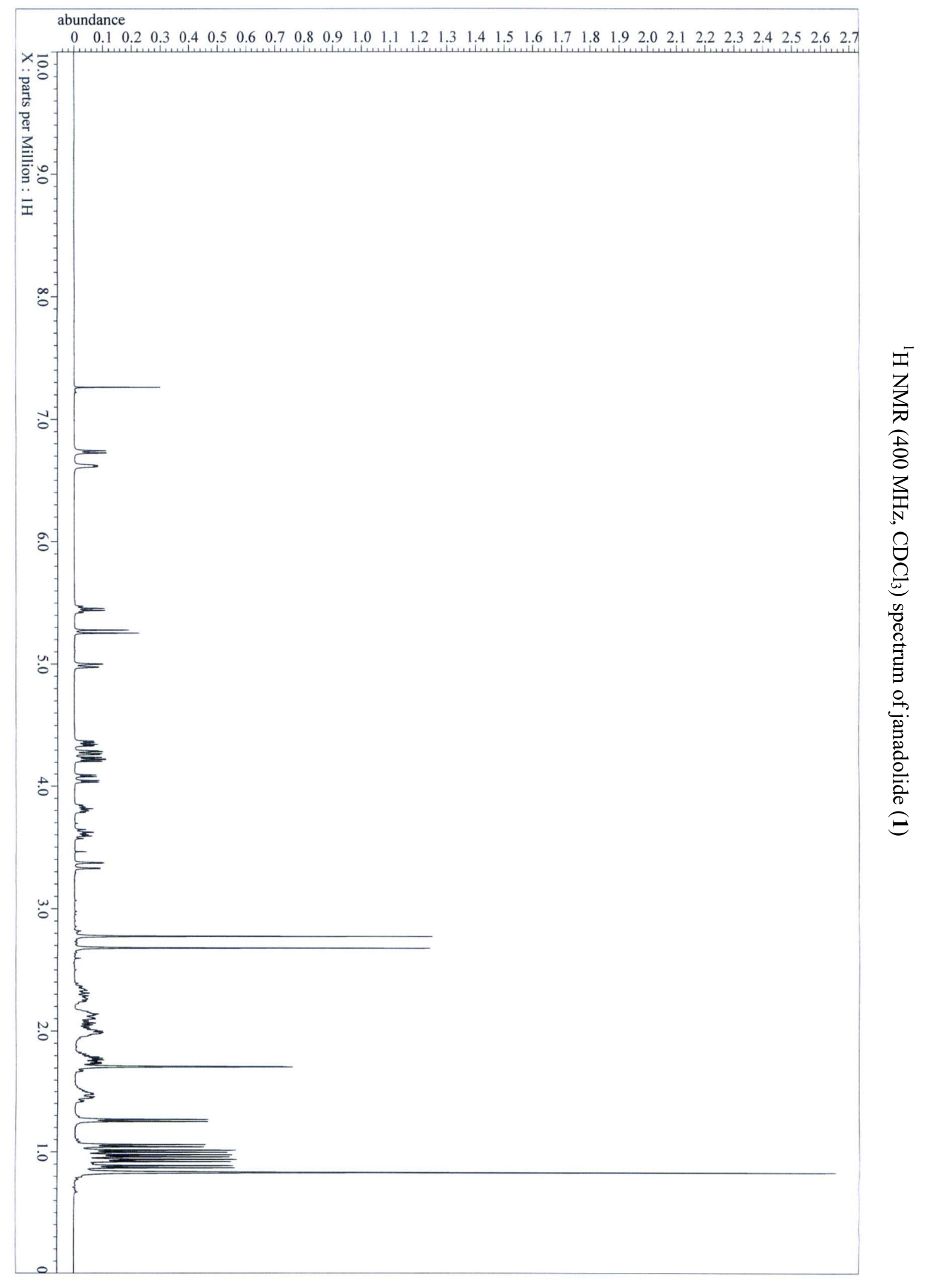




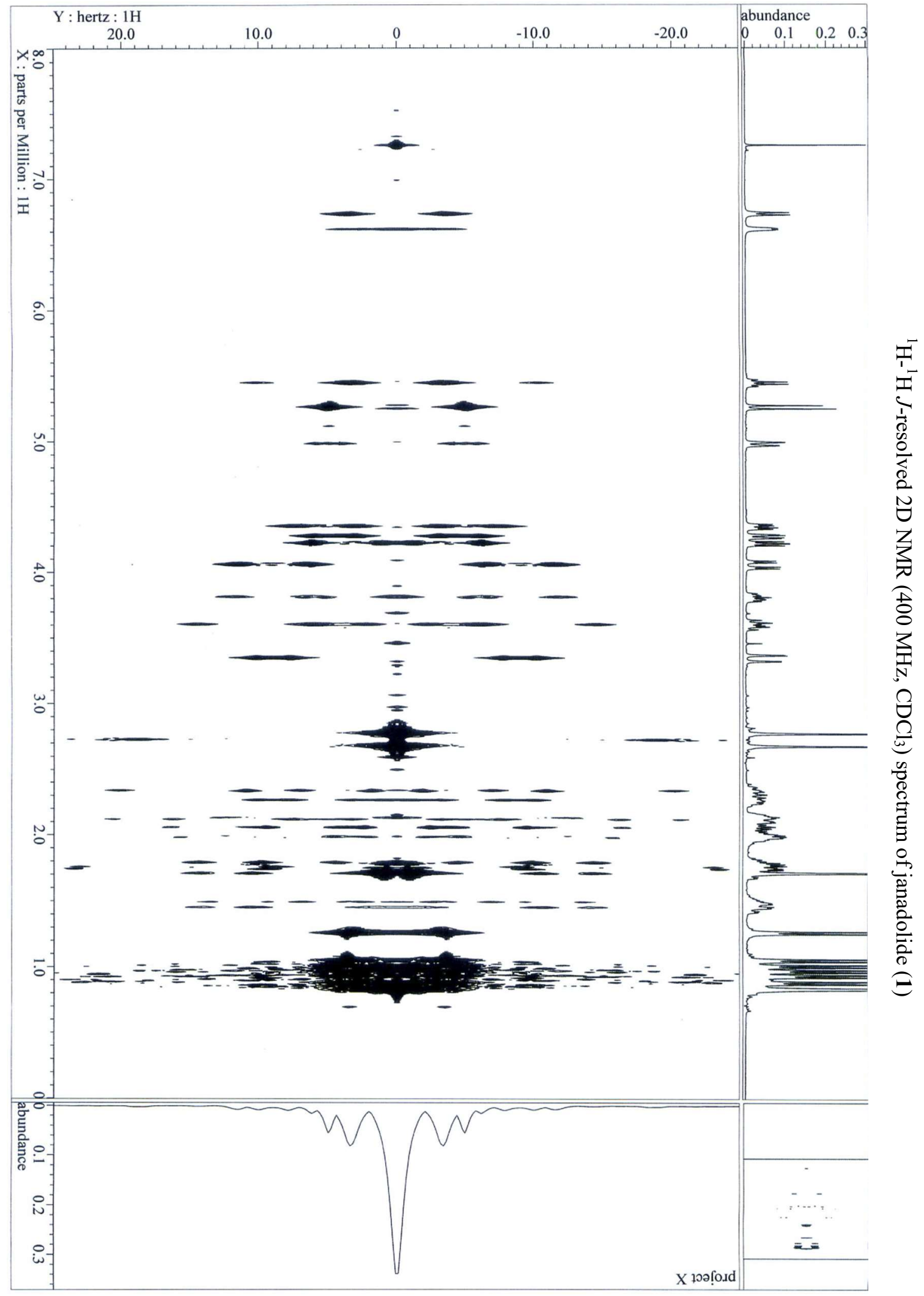




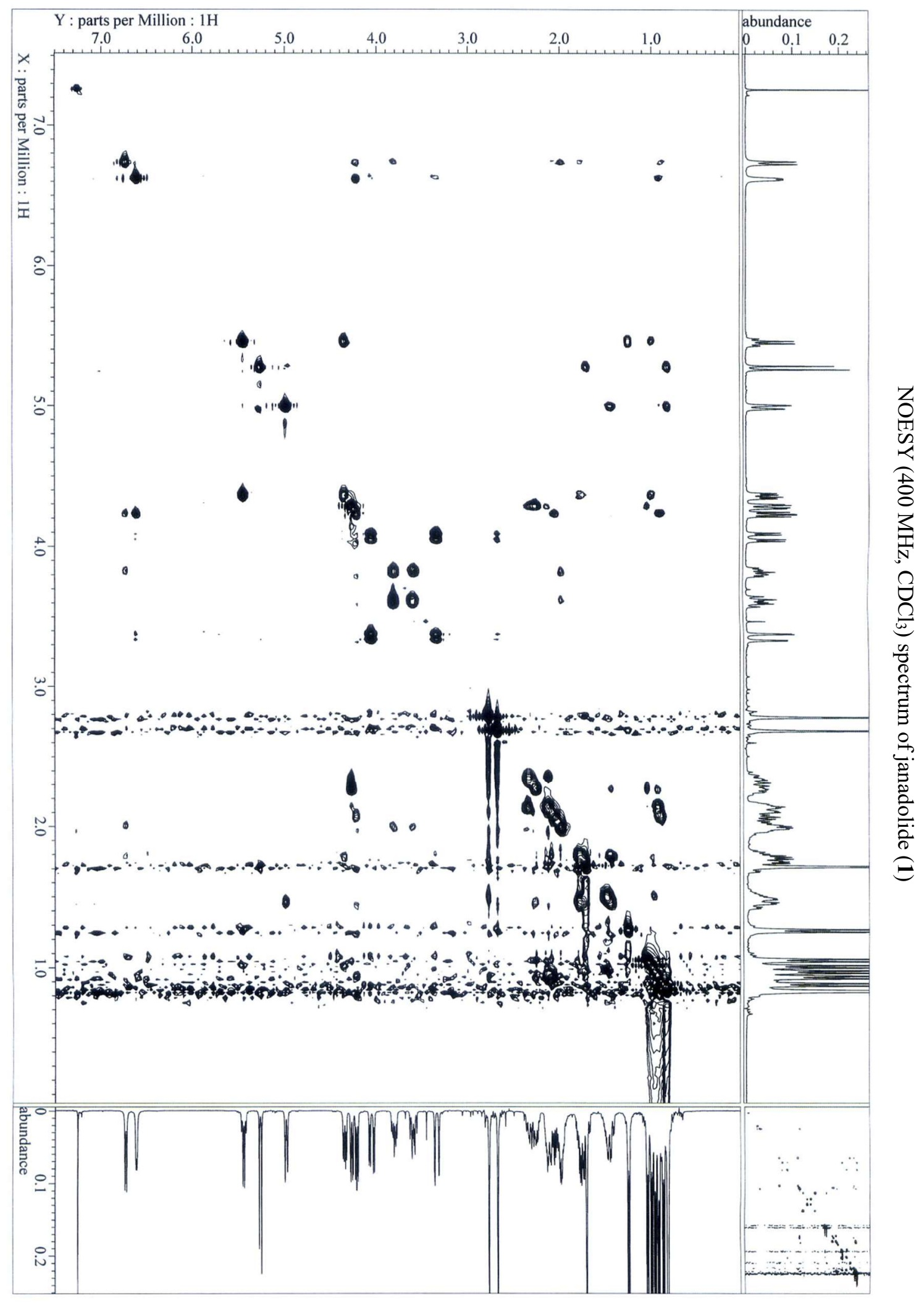




\section{Molecular modeling based on NMR data}

We preliminarily investigated the lowest energy conformation of janadolide (1) in the same way as Doi et al. ${ }^{1}$. To determine the constraints, the NMR data of $\mathbf{1}$ in $\mathrm{CDCl}_{3}$ was analyzed. ${ }^{3} J_{\mathrm{H}, \mathrm{H}}$ values were determined by ${ }^{1} \mathrm{H}$ NMR and ${ }^{1} \mathrm{H}-{ }^{1} \mathrm{H} J$-resolved 2D NMR spectra. Vicinal protons which were anti-oriented $\left({ }^{3} J_{\mathrm{H}, \mathrm{H}} \geq 10 \mathrm{~Hz}\right)$ were interpreted as dihedral angle constraints. NOESY experiments performed at a mixing time of $500 \mathrm{~ms}$ and gave ${ }^{1} \mathrm{H}-{ }^{1} \mathrm{H}$ internuclear distances. To determine distance constraints, the cross peaks of the NOESY spectrum were translated into three distance constraint categories (strong, $\leq 2.5 \AA$; medium, $\leq 3.5 \AA$; and weak, $\leq 5.0 \AA$ ) depending on their signal intensities.

Molecular modeling was performed on the MacroModel (version 10.4) program $^{2-4}$ by the distance geometry method. We utilized an OPLS-2005 force field and a generalized Born/solvent-accessible surface area (GB/SA) solvent model. ${ }^{5}$ The calculations were conducted in a chloroform environment. To find 3D structures that were in agreement with the experimental data and had low energies in a given field, we selected a protocol that comprised two steps. First, a conformational search was conducted using Monte Carlo-based torsional sampling with ${ }^{1} \mathrm{H}-{ }^{1} \mathrm{H}$ distance constraints (force constant, $\left.10 \mathrm{~kJ} \mathrm{~mol}^{-1} \AA^{-2}\right)$ and anti-oriented dihedral constraints $\left({ }^{1} \mathrm{H}-\mathrm{C}-\mathrm{C}-{ }^{1} \mathrm{H}\right.$ angle, $\left.180 \pm 30^{\circ}\right)$ at 10,000 iterations with 500 times of energy minimization. Then, energy minimization was performed 5,000 times on each found structure without constraints.

As a result, the lowest energy conformation of janadolide (1) was revealed as shown in Figure S1B. In these conformations, two amide bonds between $N$-Me-Leu and $N$-Me-Ala and between Pro and 
the polyketide moiety were found to be s-cis. This result was also supported by the difference of the $\beta$ and $\gamma$ carbon chemical shifts $(8.9 \mathrm{ppm}){ }^{6}$

These results will be useful information for designing the synthetic analogues of janadolide which show the more potent anti-trypanosomal activity.
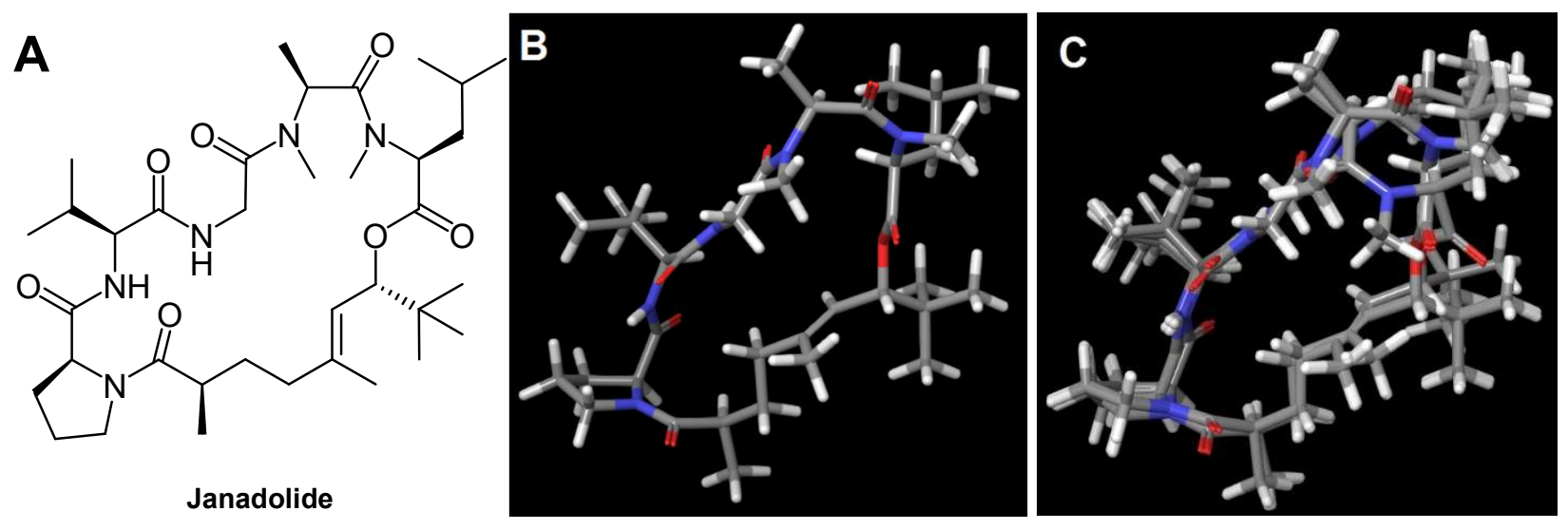

Figure S1. Molecular modeling of janadolide (1) in chloroform environment. (A) 2D structure of janadolide, (B) the lowest energy conformation of janadolide, and (C) superposition of top 5 stable conformations, representing $99.8 \%$ of the total Boltzmann distribution of conformers. 
Table S2. Detailed NMR spectral data of janadolide (1) in $\mathrm{CDCl}_{3}$

\begin{tabular}{|c|c|c|c|}
\hline Unit & position & $\delta c^{a}$ & $\delta_{\mathrm{H}^{\mathrm{b}}}^{\mathrm{b}}(J$ in $\mathrm{Hz})$ \\
\hline \multirow[t]{8}{*}{ Pro } & 1 & $172.8, \mathrm{C}$ & \\
\hline & 2 & $62.1, \mathrm{CH}$ & $4.28, \mathrm{dd}(9.1,2.6)$ \\
\hline & $3 a$ & $32.0, \mathrm{CH}_{2}$ & $2.34, \mathrm{~m}$ \\
\hline & $3 b$ & & $2.14, \mathrm{~m}$ \\
\hline & $4 \mathrm{a}$ & $23.1, \mathrm{CH}_{2}$ & $2.00, \mathrm{~m}$ \\
\hline & $4 \mathrm{~b}$ & & $1.99, \mathrm{~m}$ \\
\hline & $5 \mathrm{a}$ & $46.8, \mathrm{CH}_{2}$ & 3.82, ddd $(12.3,5.0,4.5)$ \\
\hline & $5 b$ & & 3.61, ddd $(12.3,8.8,7.7)$ \\
\hline \multirow[t]{6}{*}{ Val } & 1 & 169.1, C & \\
\hline & 2 & $58.1, \mathrm{CH}$ & $4.23, \mathrm{dd}(7.3,5.3)$ \\
\hline & 3 & $32.7, \mathrm{CH}$ & $2.06, \mathrm{~m}$ \\
\hline & 4 & $18.73, \mathrm{CH}_{3}$ & $0.93, d(7.0)$ \\
\hline & 5 & $18.71, \mathrm{CH}_{3}$ & $0.88, \mathrm{~d}(7.0)$ \\
\hline & $\mathrm{NH}$ & & $6.73, \mathrm{~d}(5.3)$ \\
\hline \multirow[t]{4}{*}{ Gly } & 1 & 166.9, C & \\
\hline & $2 \mathrm{a}$ & $41.3, \mathrm{CH}_{2}$ & 4.06, dd $(18.3,5.2)$ \\
\hline & $2 b$ & & $3.35, \mathrm{dd}(18.3,1.9)$ \\
\hline & $\mathrm{NH}$ & & $6.62, \mathrm{dd}(5.2,1.9)$ \\
\hline \multirow[t]{4}{*}{$N$-Me-Ala } & 1 & $170.43, \mathrm{C}$ & \\
\hline & 2 & $49.7, \mathrm{CH}$ & $5.45, \mathrm{q}(6.8)$ \\
\hline & 3 & $14.7, \mathrm{CH}_{3}$ & $1.26, \mathrm{~d}(6.8)$ \\
\hline & $N$-Me & $29.1, \mathrm{CH}_{3}$ & $2.68, \mathrm{~s}$ \\
\hline \multirow[t]{8}{*}{$N$-Me-Leu } & 1 & 170.41, C & \\
\hline & 2 & $58.2, \mathrm{CH}$ & $4.35, \mathrm{dd}(10.4,4.5)$ \\
\hline & $3 a$ & 38.6, $\mathrm{CH}_{2}$ & $1.81, \mathrm{~m}$ \\
\hline & $3 b$ & & $1.73, \mathrm{~m}$ \\
\hline & 4 & $25.0, \mathrm{CH}$ & $1.47, \mathrm{~m}$ \\
\hline & 5 & $23.5, \mathrm{CH}_{3}$ & $0.97, \mathrm{~d}(6.7)$ \\
\hline & 6 & $21.6, \mathrm{CH}_{3}$ & $1.00, \mathrm{~d}(6.6)$ \\
\hline & $N-\mathrm{Me}$ & $30.0, \mathrm{CH}_{3}$ & $2.78, \mathrm{~s}$ \\
\hline \multirow[t]{13}{*}{ Polyketide moiety } & 1 & 175.9, C & \\
\hline & 2 & $39.5, \mathrm{CH}$ & $2.29, \operatorname{ddq}(10.9,6.8,2.1)$ \\
\hline & $3 \mathrm{a}$ & $35.3, \mathrm{CH}_{2}$ & 2.11, dddd $(16.0,12.2,10.9,4.9)$ \\
\hline & $3 b$ & & $0.95, \mathrm{~m}$ \\
\hline & $4 a$ & $40.0, \mathrm{CH}_{2}$ & 1.77, ddd $(12.1,10.0,4.9)$ \\
\hline & $4 \mathrm{~b}$ & & 1.45, ddd $(12.2,12.1,4.0)$ \\
\hline & 5 & $143.5, \mathrm{C}$ & \\
\hline & 6 & $119.9, \mathrm{CH}$ & $4.99, \mathrm{dq}(10.0,0.9)$ \\
\hline & 7 & $79.5, \mathrm{CH}$ & $5.27, \mathrm{~d}(10.0)$ \\
\hline & 8 & $35.9, \mathrm{C}$ & \\
\hline & 9 & $25.8, \mathrm{CH}_{3} \times 3$ & $0.83, \mathrm{~s}$ \\
\hline & 10 & $19.3, \mathrm{CH}_{3}$ & $1.05, \mathrm{~d}(6.8)$ \\
\hline & 11 & $16.8, \mathrm{CH}_{3}$ & $1.71, \mathrm{~d}(0.9)$ \\
\hline
\end{tabular}

${ }^{\mathrm{a}}$ Measured at $100 \mathrm{MHz} .{ }^{\mathrm{b}}$ Measured at $400 \mathrm{MHz}$. 
Table S3. Distance and dihedral angle costraints for molecular modeling of janadolide (1).

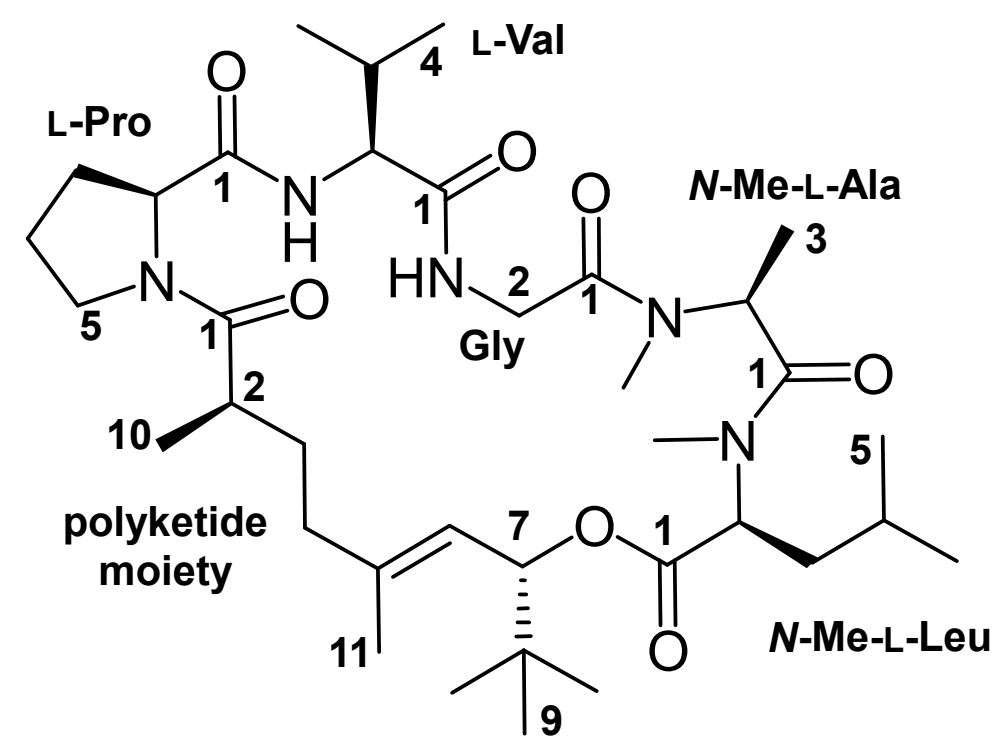

Janadolide (1)

\begin{tabular}{|c|c|c|c|}
\hline $\begin{array}{l}\text { Dihedral Angle } \\
\qquad\left(180^{\circ} \pm 30^{\circ}\right)\end{array}$ & $\begin{array}{l}\text { Strong } \\
(\sim 2.5 \AA)\end{array}$ & $\begin{array}{l}\text { Medium } \\
(\sim 3.5 \AA)\end{array}$ & $\begin{array}{c}\text { Weak } \\
(\sim 5.0 \AA)\end{array}$ \\
\hline $\mathrm{H}_{2}-\mathrm{C}_{2}-\mathrm{C}_{3}-\mathrm{H}_{3 \mathrm{a}}$ (polyketide) & $\mathrm{H}_{2}(N-\mathrm{Me}-\mathrm{Ala})-\mathrm{H}_{2}(N-\mathrm{Me}-\mathrm{Leu})$ & $\mathrm{H}_{3}(N-\mathrm{Me}-\mathrm{Ala})-\mathrm{H}_{5 / 6}(N-\mathrm{Me}-\mathrm{Leu})$ & $\mathrm{H}_{2}$ (polyketide) $-\mathrm{H}_{4 \mathrm{~b}}($ polyketide $)$ \\
\hline $\mathrm{H}_{3 b}-\mathrm{C}_{3}-\mathrm{C}_{4}-\mathrm{H}_{4 a}$ (polyketide) & $\mathrm{H}_{7}$ (polyketide)- $\mathrm{H}_{11}$ (polyketide) & $\mathrm{H}_{2}(N-\mathrm{Me}-\mathrm{Leu})-\mathrm{H}_{6}(N-\mathrm{Me}-\mathrm{Leu})$ & $\mathrm{H}_{2 \mathrm{~b}}(\mathrm{Gly})-N-\mathrm{Me}(N$-Me-Ala $)$ \\
\hline $\mathrm{H}_{3 \mathrm{a}}-\mathrm{C}_{3}-\mathrm{C}_{4}-\mathrm{H}_{4 b}($ polyketide $)$ & $\mathrm{H}_{2}$ (Pro)- $\mathrm{H}_{2}$ (polyketide) & $\mathrm{H}_{6}($ polyketide $)-\mathrm{H}_{4 b}($ polyketide $)$ & $\mathrm{NH}(\mathrm{Gly})-\mathrm{H}_{4 / 5}(\mathrm{Val})$ \\
\hline \multirow[t]{5}{*}{$\mathrm{H}_{6}-\mathrm{C}_{6}-\mathrm{C}_{7}-\mathrm{H}_{7}$ (polyketide) } & $\mathrm{NH}(\mathrm{Gly})-\mathrm{H}_{2}$ (Val) & $\mathrm{H}_{6}$ (polyketide)-H9(polyketide) & $\mathrm{NH}(\mathrm{Val})-\mathrm{H}_{4 a}$ (polyketide) \\
\hline & & $\mathrm{H}_{2 \mathrm{a}}(\mathrm{Gly})-N-\mathrm{Me}(N-\mathrm{Me}-\mathrm{Ala})$ & \\
\hline & & $\mathrm{H}_{2}$ (polyketide)- $\mathrm{H}_{3 \mathrm{~b}}($ polyketide) & \\
\hline & & $\mathrm{H}_{2}$ (Pro)- $\mathrm{H}_{10}($ polyketide $)$ & \\
\hline & & $\mathrm{NH}\left(\right.$ Val)-H $\mathrm{H}_{5 \mathrm{a}}($ Pro $)$ & \\
\hline
\end{tabular}




\section{Evaluation of the biological activity of janadolide (1)}

To evaluate the growth-inhibitory activities of janadolide (1), an MTT assay was conducted with HeLa and HL60 cells. The cells were treated in 96-well plates with various concentrations of janadolide (10-100 $\mu \mathrm{M}$ for each cell type) for $72 \mathrm{~h}$. These assays revealed that janadolide did not inhibit the growth of HL60 and HeLa cells ( $\mathrm{IC}_{50} 19 \pm 5 \mu \mathrm{M}$ and $>100 \mu \mathrm{M}$, respectively).

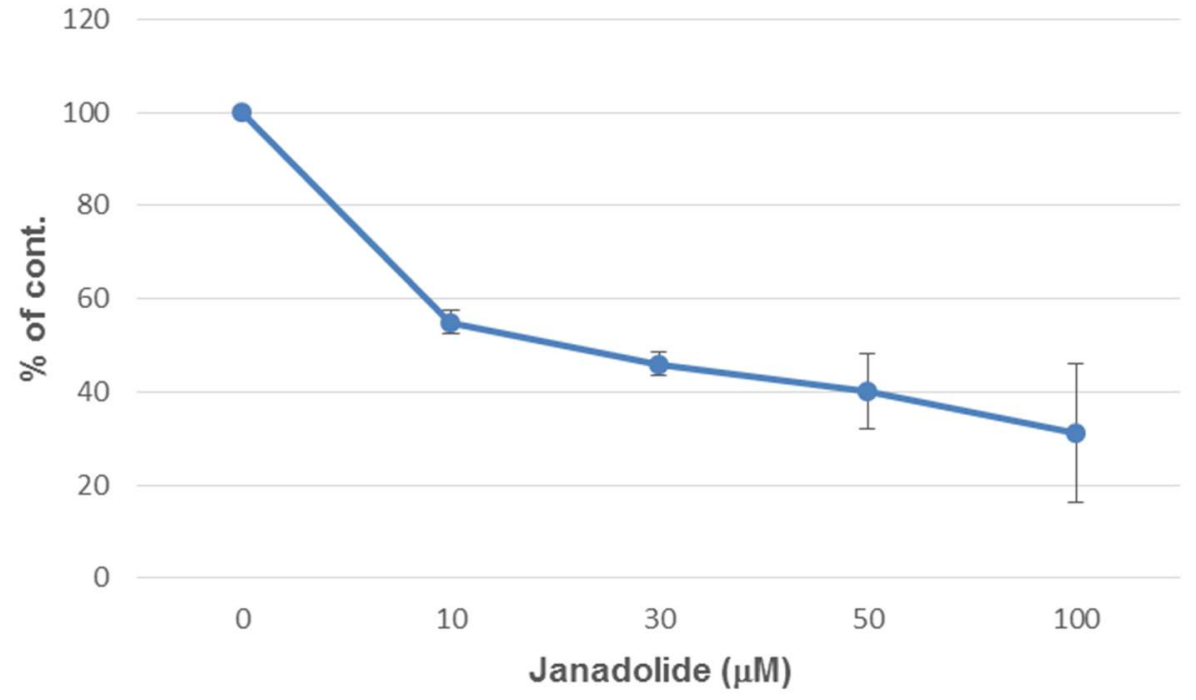

Figure S2. The original curved with $\mathrm{IC}_{50}$ determination against HL60 cells $(\mathrm{n}=3)$. 


\section{Identification of the marine cyanobacterium}

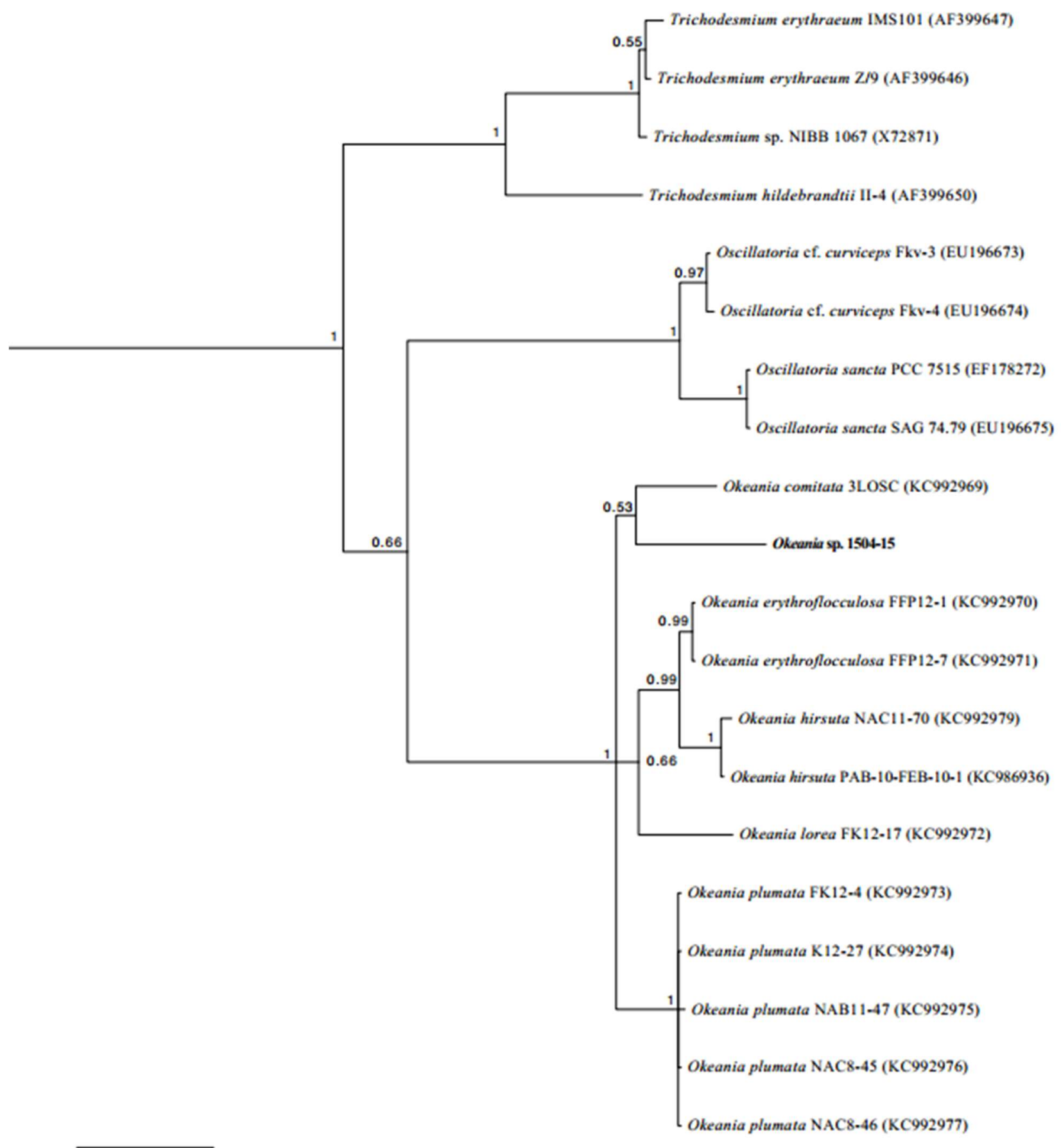

0.07

Figure S3. Phylogenetic inference of the Okeania specimens based on the 16S-23S internal transcribed spacer (ITS) region. The phylogenetic analysis was conducted in the same way as Ellisman et al. ${ }^{7}$. The evolutionarily distant Moorea producens $3 \mathrm{~L}$ and M. bouillonii PNG5-198 were included as out-groups. 


\section{Reference for the supporting information}

(1) Masuda, Y.; Suzuki, J.; Onda, Y.; Fujino, Y.; Yoshida, M.; Doi, T. J. Org. Chem. 2014, 79, 8000-8009.

(2) Mohamadi, F.; Richards, N. G. J.; Guida, W. C.; Liskamp, R.; Lipton, M.; Caufield, C.; Chang, G.; Hendrickson, T.; Still, W. C. J. Comput. Chem. 1990, 11, 440-467.

(3) Still, W. C.; Tempczyk, A.; Hawley, R. C.; Hendrickson, T. J. Am. Chem. Soc. 1990, 112, 6127-6129.

(4) Schrödinger Suite 2014: MacroModel, version 10.4; Schrödinger, LLC: New York, 2014.

(5) Qiu, D.; Shenkin, P. S.; Hollinger, F. P.; Still, W. C. J. Phys. Chem. A 1997, 101, 3005-3014.

(6) Schubert, M.; Labudde, D.; Oschkinat, H.; Schmieder, P. J. Biomol. NMR 2002, 24, 149-154.

(7) Engene, N.; Paul, V. J.; Byrum, T.; Gerwick, W. H.; Thor, A.; Ellisman, M. K. J. Phycol. 2013, 49, 1095-1106. 